\title{
Environmental Monitoring Around an Offshore Fish Farm with Copper Alloy Mesh Pens in the Northern Aegean Sea
}

\author{
Yesim Buyukates ${ }^{1}$, Barbaros Celikkol ${ }^{2}$, Murat Yigit ${ }^{3,}$, , Judson DeCew ${ }^{4}$, Musa Bulut ${ }^{3}$ \\ ${ }^{1}$ Department of Marine Biology, Faculty of Marine Science and Technology, Canakkale Onsekiz Mart University, Canakkale, Turkey \\ ${ }^{2}$ Department of Mechanical and Ocean Engineering, University of New Hampshire, Durham, NH - USA \\ ${ }^{3}$ Department of Aquaculture and Marine Technology, Faculty of Marine Science and Technology, Canakkale Onsekiz Mart University, \\ Canakkale, Turkey \\ ${ }^{4}$ Jere A. Chase Ocean Engineering Laboratory, School of Marine Science and Ocean Engineering, University of New Hampshire, Durham, \\ $\mathrm{NH}-\mathrm{USA}$
}

\section{Email address: \\ muratyigit@comu.edu.tr (M. Yigit) \\ ${ }^{*}$ Corresponding author}

\section{To cite this article:}

Yesim Buyukates, Barbaros Celikkol, Murat Yigit, Judson DeCew, Musa Bulut. Environmental Monitoring Around an Offshore Fish Farm with Copper Alloy Mesh Pens in the Northern Aegean Sea. American Journal of Environmental Protection. Vol. 6, No. 2, 2017 , pp. 50-61. doi: 10.11648/j.ajep.20170602.13

Received: March 10, 2017; Accepted: March 25, 2017; Published: April 10, 2017

\begin{abstract}
In the present study, environmental monitoring was undertaken around an offshore fish farm with copper alloy mesh pens located in the Northern Aegean Sea. From the water velocity results obtained at the farm site, located in the Strait of Canakkale (formerly the Dardanelles) it was recorded that the horizontal water velocity reached $36 \mathrm{cms}^{-1}$ at select times. The average water velocity was found to be $22 \mathrm{cms}^{-1}$. Water quality measurements were made over an 11 months period between September 2011 and July 2012. Other than occasional wind drawn mixing events, the two different water masses can be clearly indicated depending on the temperature and salinity values recorded throughout the study period in the Strait of Canakkale. Inorganic nutrient concentrations such as $\mathrm{NO}_{2}^{-}+\mathrm{NO}_{3}^{-}, \mathrm{NH}_{4}, \mathrm{PO}_{4}, \mathrm{TP}$ and $\mathrm{SiO}_{2}$, changed from low to moderate through most of the sampling period. The data for TSS varied among sampling depths between 0.06 and $10.40 \mathrm{mgL}^{-1}$, being below the typical seawater quality measurement threshold of $30 \mathrm{mgL}^{-1}$ pronounced by Water Pollution Control Regulations. Chlorophyll- $a$ concentration, ranged between $0.143-2.633 \mu \mathrm{gL}^{-1}, 0.055-1.519 \mu \mathrm{gL}^{-1}$, and $0.110-2.288 \mu \mathrm{gL}^{-1}$, at the surface, $15 \mathrm{~m}$, and $30 \mathrm{~m}$, respectively. It has been observed that starting from March 2012, especially in Stations 2 and 3, the phytoplankton abundance controlled the TSS, rather than terrestrial sources.
\end{abstract}

Keywords: Aegean Sea, Copper Alloy Mesh Cage, Environmental Parameters

\section{Introduction}

Organic matter growth on marine systems is one of the challenges for the cage aquaculture industry. Biofouling on the nettings causes problems in terms of blockage of water flow through the net mesh, which then decreases oxygen concentration in the water column. Any new production method or material for the reduction of biofouling on cage systems might bring important benefits in terms of better growth performance of fish by better feed intake, reduced stress conditions and less labor costs due to net changes or cleaning [1]. The utilization and benefits of copper, with its antimicrobial properties, have been well documented in health care applications [2]. Nowadays, copper alloys can be found as wire mesh materials that can be used in cage nets instead of polymer nettings. Copper alloy mesh has been reported as a biofouling resistant material for fish culture in the Mediterranean, with improved fish health under better sanitary conditions in a cleaner cage environment [1]. Furthermore, copper alloy meshes demonstrated improved economic benefits when compared to traditional nylon nettings for the Atlantic salmon aquaculture in Chile [3]. The drag forces on fish nets due to strong currents in the open ocean are one of the reasons for fish stress. Copper alloy 
mesh may reduce the drag forces on these nets, and improve the strength of fish cages under high-energy offshore environments [4].

Sea bream and sea bass are the most important marine species for the European aquaculture industry, especially with the high production rates from Turkey and Greece in the Mediterranean [5, 6]. Usage of traditional nylon nets, sometimes with the administration of double nets, especially in order to prevent damage by the bites of sea bream having strong and sharp teeth, is well known in the Mediterranean aquaculture. Although inexpensive, these nets are not durable enough mostly during strong weather conditions such as the research location (Canakkale Strait) experiencing approximately 10 or 12 storms each year [5]. The use of copper alloy nets in the European Seas might support the sustainable future of the aquaculture industry. Hence, the improvement that may help expand the European finfish aquaculture involves innovation in the aquaculture pens themselves. Copper alloy meshes seem to be promising material with increased profit and reduced maintenance costs and environmental concerns [7]. However, there is lack of information on variations of water quality parameter around copper alloy net cages operating in the same site over a long period. The only study available was published by [7], who reported reduced environmental performance of copper alloy mesh in terms of life cycle assessment for Atlantic salmon grow-out farm in Chile. However, any information regarding to environmental monitoring of copper alloy cages in offshore conditions with respect to bio-chemical water parameters are still missing. Hence, the aim of the present study was to monitor and evaluate variations in water quality parameters around an offshore fish farm with copper alloy mesh pens.

\section{Materials and Methods}

The water velocity at the farm site, located in the Canakkale Strait was obtained over a 27 hour period between May 29-30, 2012 (Figure 1). Longer sampling was not required due to the steady water flow from the Black Sea to the Aegean Sea (surface) and a reverse flow (subsurface). Tides in the area are on the order of $30 \mathrm{~cm}$, therefore do not influence the water velocity magnitudes or directions. A current-meter $(1.000 \mathrm{kHz}$ Aanderaa RDCP) was used to monitor the current direction and velocity. The RDCP utilizes sound to monitor particulates in the water column (traveling with the incident flow) to obtain velocity and direction. The RDCP was secured to a buoy at the site, 2 meters below the sea surface in a down-looking mode. This orientation was preferred to an upward-looking bottom mounting as no loss of data near the surface would occur (due to side lobes interacting with the water surface). The water velocities were sampled every 10-minutes, in order to record most accurate data due to the frequent differentiation of the two way main current directions in the Strait of Canakkale.

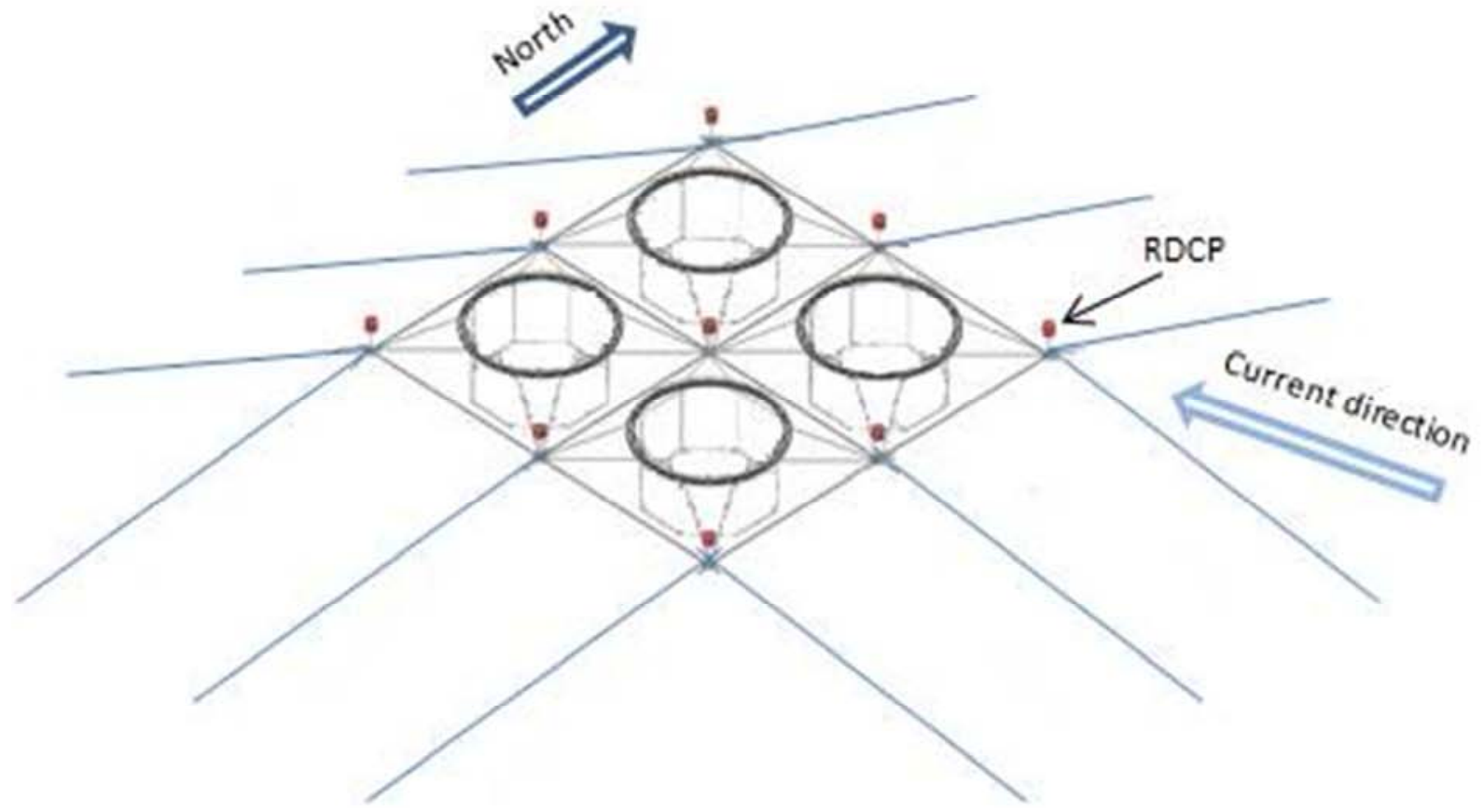

Figure 1. Schematic of the $2 \times 2$ bay grid-mooring system with copper-alloy mesh cages (top view and side view). The RDCP (• current-meter, $1.000 \mathrm{kHz}$ Aanderaa) was placed adjacent to the net pens.

Water quality measurements were made over an 11 months period between September 2011 and July 2012. To assess the water quality at and around the farm site, water samples were obtained from three stations, as seen in Figure2:

Station 1 - net pens (surface and $5 \mathrm{~m}$ )
Station 2 - distance of 25 meters downstream from net pens (surface, 15 and $30 \mathrm{~m}$ )

Station 3 - distance of 15 meters upstream from net pens (surface, 15 and $30 \mathrm{~m}$ ) 


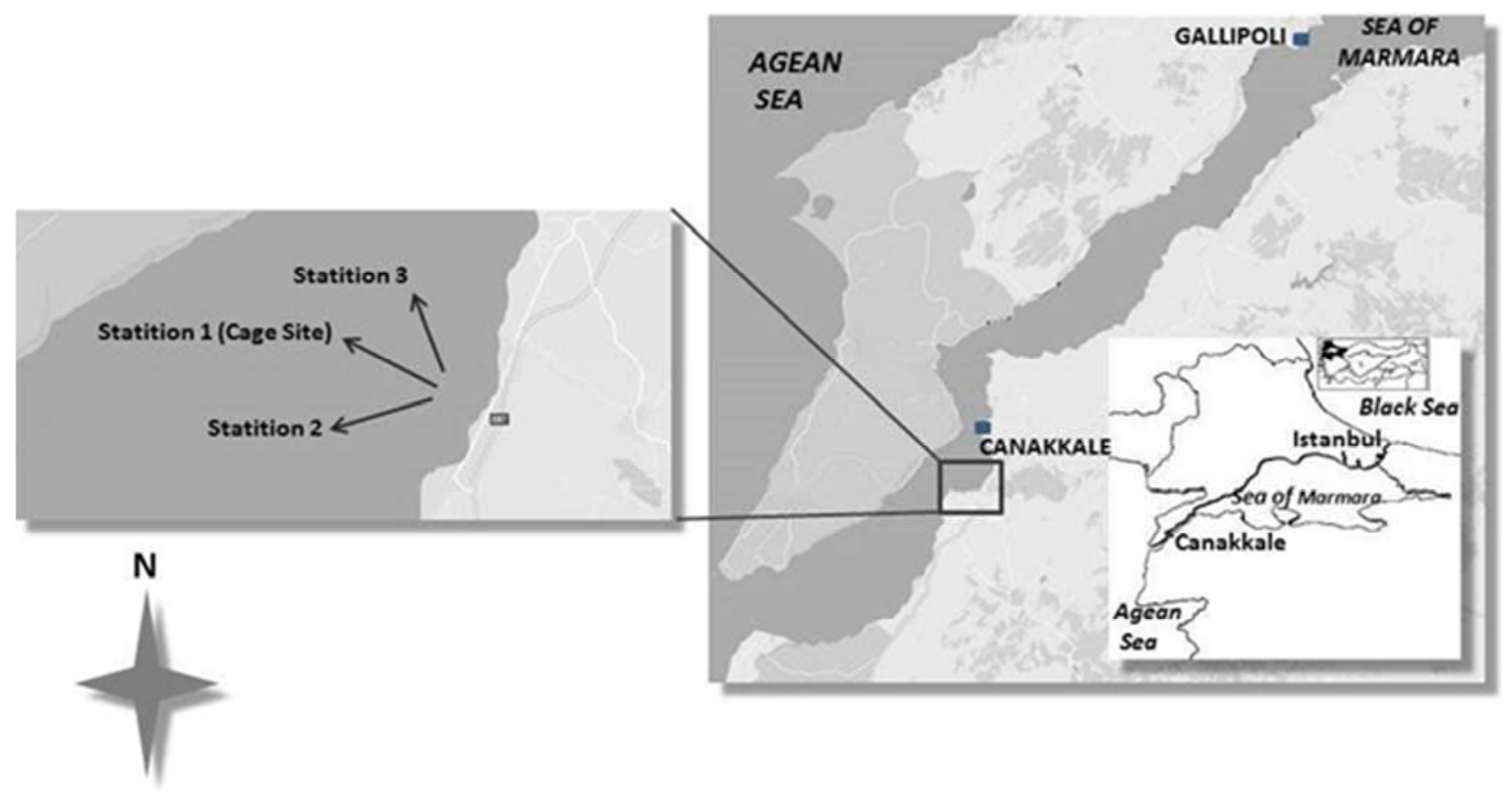

Figure 2. Location of the study area with three sampling stations.

Water quality parameters, for example, temperature, salinity, dissolved oxygen, $\mathrm{pH}$, nutrient content, chlorophyll$a$ (chl- $a$ ) and total suspended solids (TSS) were obtained. Water quality parameters were measured in situ using an YSI 600 XL MPS. Winkler Method was used to measure dissolved oxygen (DO). A $5 \mathrm{~L}$ water sampler was used to collect water samples for nutrients. Nutrient samples were then filtered through $47 \mathrm{~mm} \mathrm{GF} / \mathrm{F}$ filters by gentle vacuum and frozen for further analysis. Spectrophotometric determination of nitrite + nitrate $\left(\mathrm{NO}_{2}^{-}+\mathrm{NO}_{3}^{-}\right)$, and ammonia $\left(\mathrm{NH}_{4}\right)$ were conducted according to Strickland and Parsons (1972). Analysis for soluble reactive phosphorus $\left(\mathrm{PO}_{4}\right)$, total phosphorus (TP) and silicate $\left(\mathrm{SiO}_{2}\right)$ were conducted spectrophotometrically [8]. TSSs were analyzed gravimetrically according to [9]. GF/F filters that were used for the filtration of water samples for the nutrient analysis were wrapped in aluminum foil and kept frozen until chl- $a$ analysis was conducted. Spectrophotometric determination of chl- $a$ concentration was performed by $90 \%$ acetone extraction [10].

Finally, in order to identify meaningful relations among variables if any, various data groups were subjected to nonparametric Spearman's correlation (SPSS 23). Data were depth integrated.

\section{Results and Discussion}

The collected water velocity data was processed to obtain the horizontal and vertical water velocities and direction. Figs. 3 through 5 present the obtained water velocity and direction as a function of depth through the top portion of the water column. For reference, the farm site water depth is 45 meters. The plots only present the top 11 meters of the water column as the net pens only have a draft of 5 meters, and a significant pycnocline was found at a depth of approximately 13 meters. Due to turbulence in the interface between higher salinity Mediterranean water and less saline Black Sea water, the data below $15 \mathrm{~m}$ is quite noisy.

The measured water quality parameters (salinity, temperature, dissolved oxygen and $\mathrm{pH}$ ) are summarized in Figs. 6, 7 and 8 for the three measurement stations. Salinity ranged between 23.30 and $38.93 \%$. Water temperature varied between $10.26^{\circ} \mathrm{C}$ and $22.97^{\circ} \mathrm{C}$ at the surface, between $12.25^{\circ} \mathrm{C}$ and $19.73^{\circ} \mathrm{C}$ at $15 \mathrm{~m}$, and between $13.24^{\circ} \mathrm{C}$ and $19.40^{\circ} \mathrm{C}$ at $30 \mathrm{~m}$. The data for DO concentrations changed slightly at the three stations among sampling depths ranging between 7.10 and $10.71 \mathrm{mg} \mathrm{L}^{-1}$, while $\mathrm{pH}$ was consistent, ranging between 8.04 and 8.49 among sampling stations and various depths. 


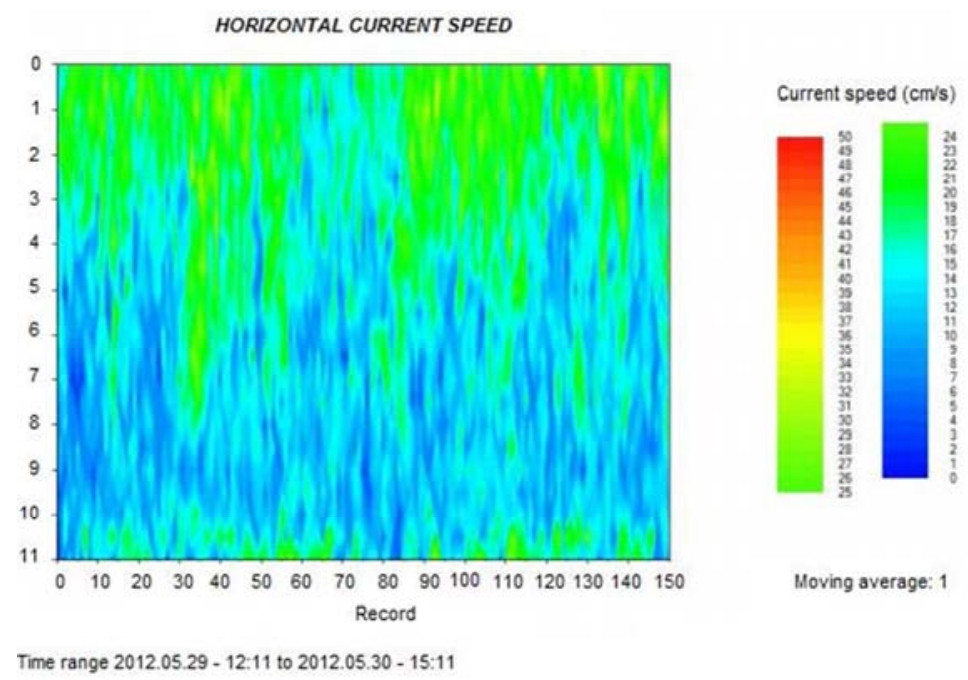

Figure 3. The surface horizontal current velocity as a function of time (records) over the 27 hour monitoring period. Water velocities were found to average around $22 \mathrm{~ms}$ near the surface, dropping to $0 \mathrm{~ms}$ at a depth of 11 meters.

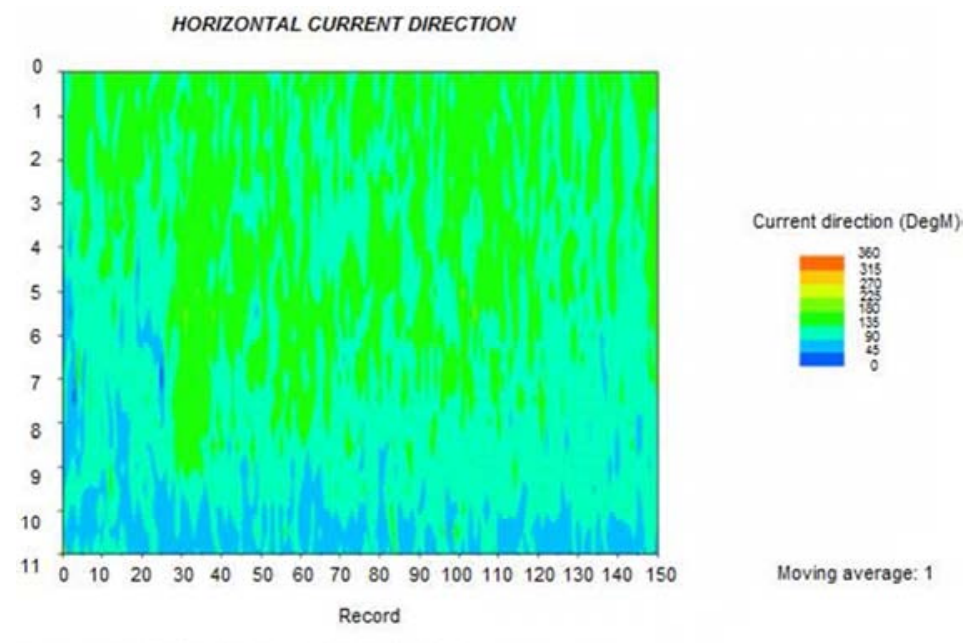

Figure 4. The current direction of the top surface layer as a function of time (records) over the 27 hour monitoring period. The data shows the water primarily heads in South towards the Aegean Sea.

VERTICAL CURRENT SPEED

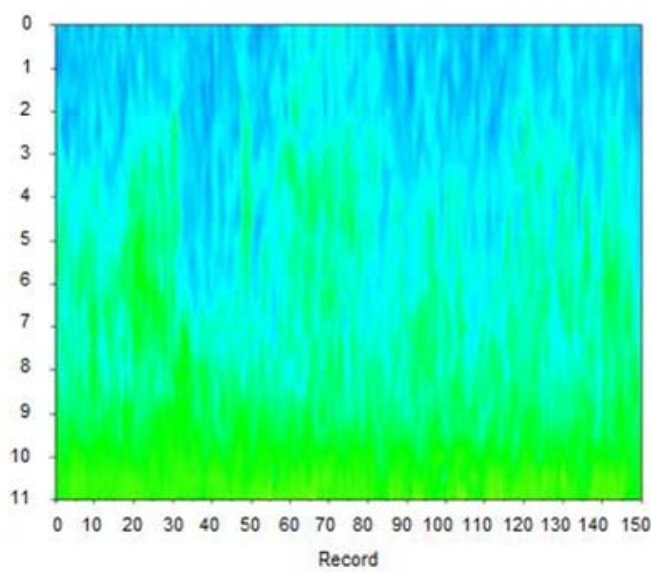

Time range: 2012.05 .29 - 12:11 to 2012.05 .30 - 15:11

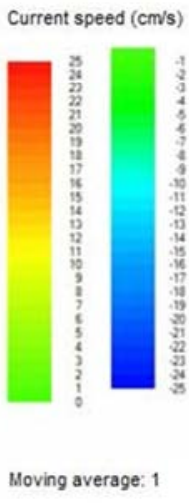

Figure 5. The vertical current velocity of the top layer of the water column as a function of time (records) over the 27 hour monitoring period. 

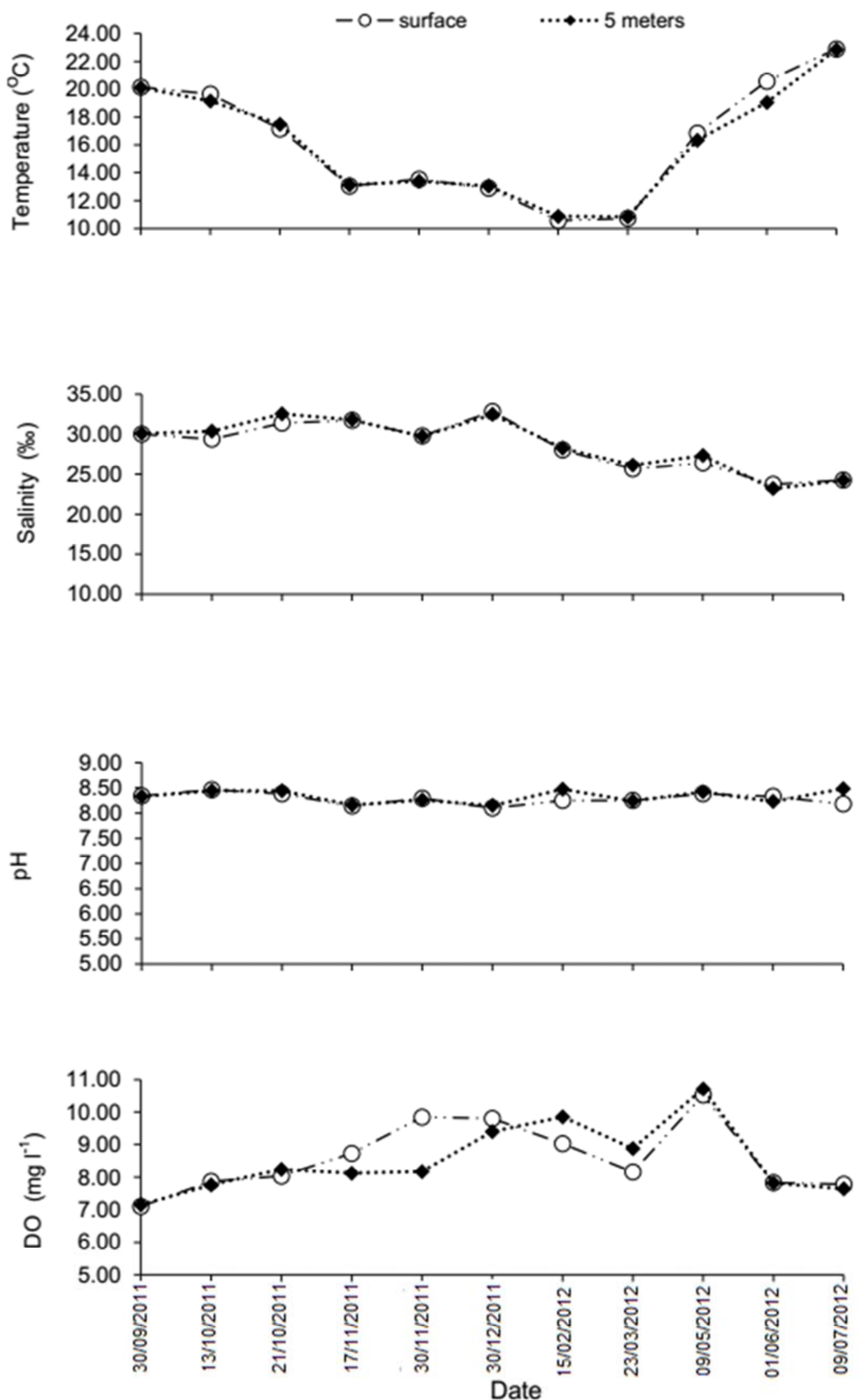

Figure 6. Temporal variations and vertical distribution of water quality parameters at the net pens (Station 1) between 30 September 2011 and 09 July 2012. 

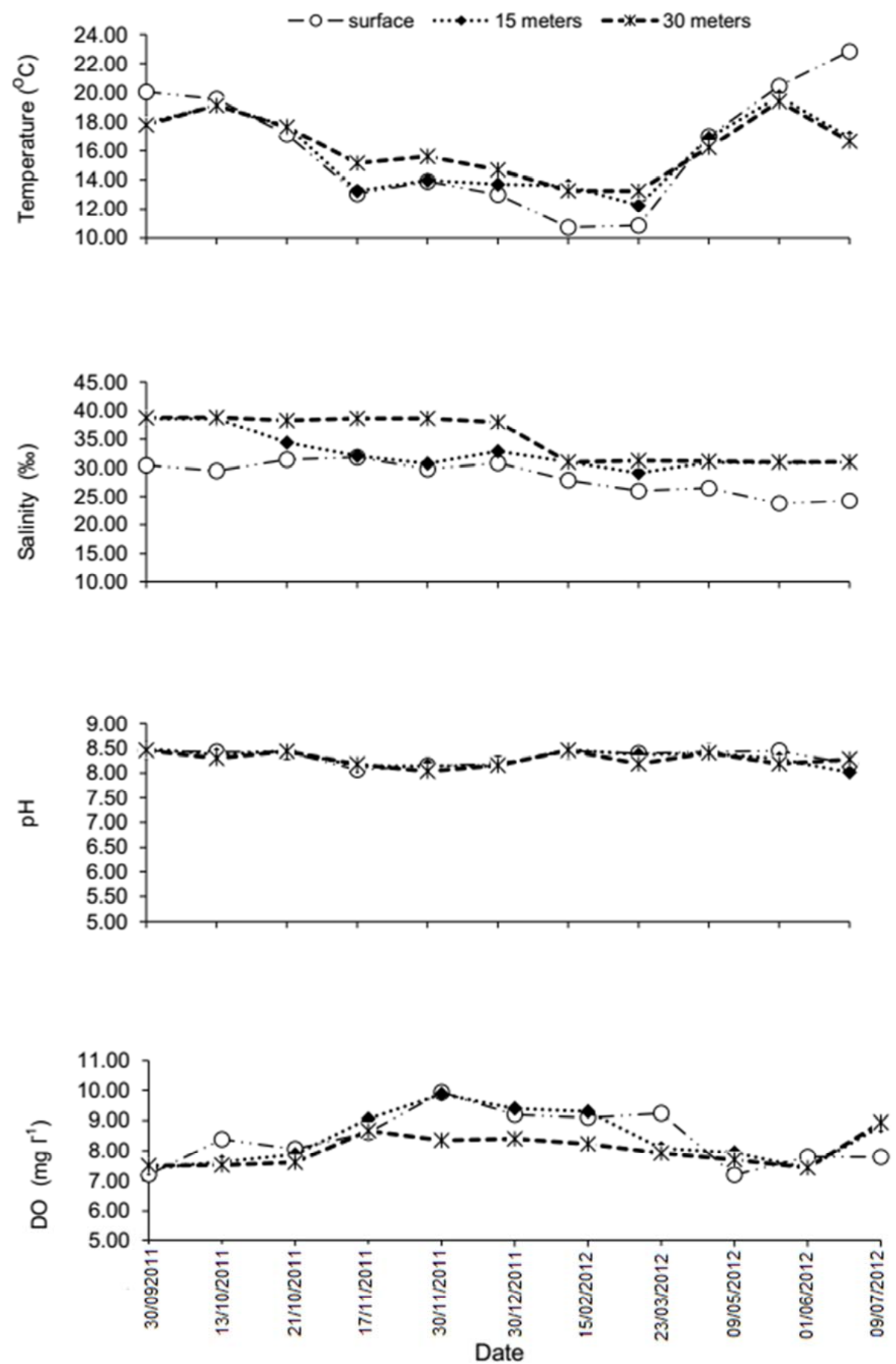

Figure 7. Temporal variations and vertical distribution of water quality parameters in Station 2 between 30 September 2011 and 09 July 2012. 

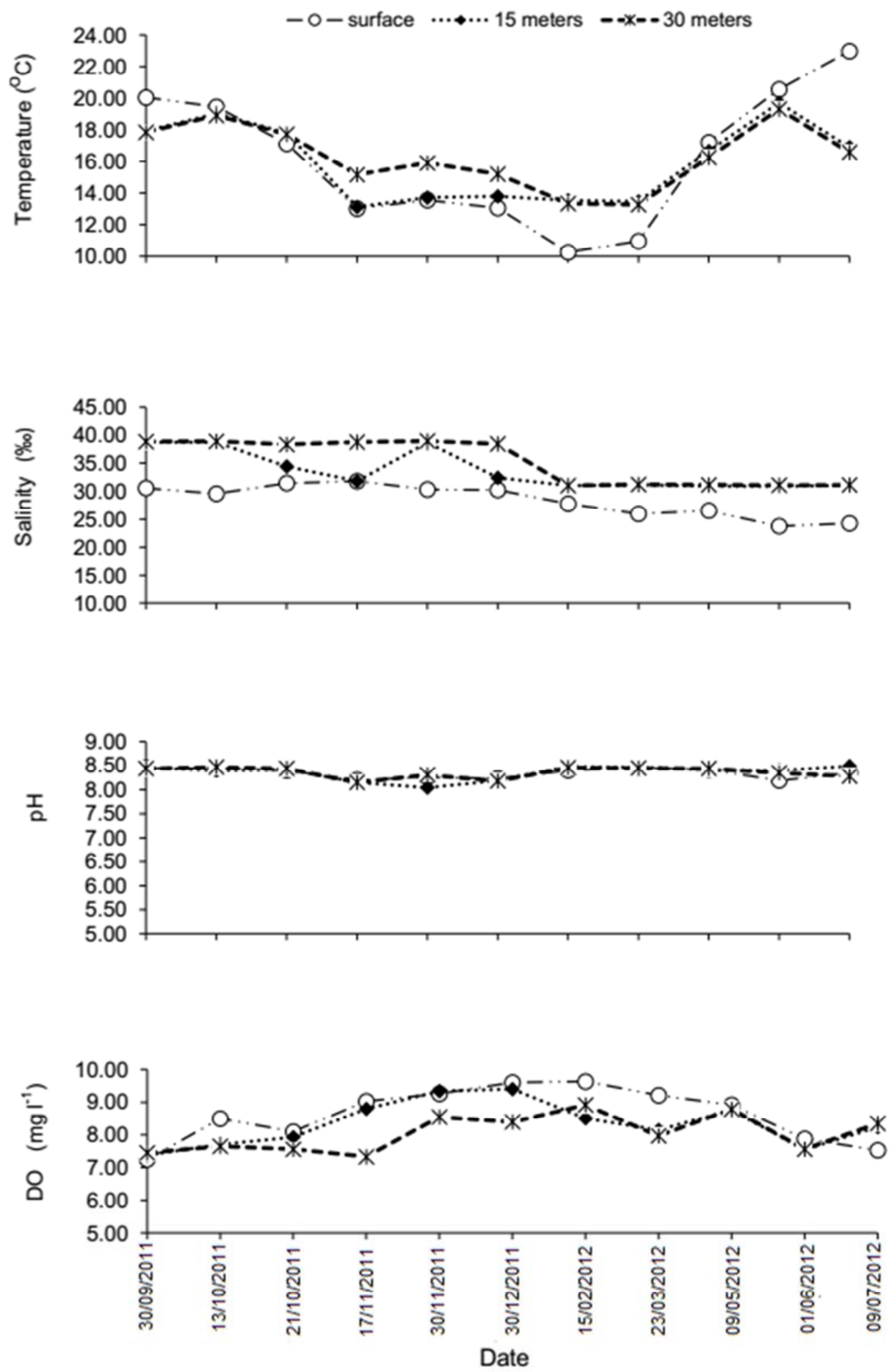

Figure 8. Temporal variations and vertical distribution of water quality parameters in Station 3 between 30 September 2011 and 09 July 2012.

The results of the inorganic nutrient concentrations are presented in Tables 1, 2 and 3 for Stations 1,2 and 3, respectively. $\mathrm{NO}_{2}^{-}+\mathrm{NO}_{3}^{-}$and $\mathrm{NH}_{4}$ varied among the sampling depths, and the sampling sites throughout the sampling periods, with values of $0.001-0.517 \mu \mathrm{mol} \mathrm{L}^{-1}$ and $0.021-2.653$ $\mu \mathrm{mol} \mathrm{L}{ }^{-1}$, respectively. $\mathrm{PO}_{4}$ changed between $0.099 \mu \mathrm{mol} \mathrm{L}-1$

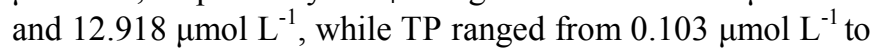
$41.837 \mu \mathrm{mol} \mathrm{L}^{-1}$ depending on the depths, the sampling sites 
and the sampling period. $\mathrm{SiO}_{2}$ ranged between 0.09 and 4.57 $\mu \mathrm{mol} \mathrm{L}^{-1}$ at the various sampling depths throughout the sampling period.

Acceptable limits reported for different physico-chemical water quality parameters [11] have been compared with those recorded in the present study for all three sampling stations around the offshore copper-alloy cage system and summarized in Table 4. Overall, water quality parameters from all three sampling stations were within the acceptable limits for fish farming throughout the sampling period in the present study.

Table 1. Temporal variations and vertical distribution of $\mathrm{NO}_{2}^{-}+\mathrm{NO}_{3}^{-} \mathrm{NH}_{4}, \mathrm{PO}_{4}, \mathrm{TP}$ and $\mathrm{SiO}_{2}$ in the Offshore Cage site (Station 1 ) between 30 September 2011 and 09 July 2012

\begin{tabular}{|c|c|c|c|c|c|c|c|c|c|c|}
\hline \multirow{2}{*}{ Date } & \multicolumn{2}{|c|}{$\mathrm{NO}_{2}^{-}+\mathrm{NO}_{3}^{-}\left(\mu \mathrm{mol} \mathrm{L}^{-1}\right)$} & \multicolumn{2}{|c|}{$\mathrm{NH}_{4}\left(\mu \mathrm{mol} \mathrm{L}^{-1}\right)$} & \multicolumn{2}{|c|}{$\mathrm{PO}_{4}\left(\mu \mathrm{mol} \mathrm{L} \mathrm{L}^{-1}\right)$} & \multicolumn{2}{|c|}{ TP $\left(\mu \mathrm{mol} \mathrm{L}^{-1}\right)$} & \multicolumn{2}{|c|}{$\mathrm{SiO}_{2}\left(\mu \mathrm{mol} \mathrm{L}{ }^{-1}\right)$} \\
\hline & Surface & $5 \mathrm{~m}$ & Surface & $5 \mathrm{~m}$ & Surface & $5 \mathrm{~m}$ & Surface & $5 \mathrm{~m}$ & Surface & $5 \mathrm{~m}$ \\
\hline 30.09 .2011 & 0.442 & 0.090 & 0.027 & 0.766 & 0.103 & 1.240 & 2.677 & 4.957 & 1.100 & 1.720 \\
\hline 13.10 .2011 & 0.033 & 0.025 & 0.086 & 2.176 & 0.103 & 0.207 & 2.181 & 4.759 & 1.080 & 2.180 \\
\hline 21.10 .2011 & 0.011 & 0.040 & 0.359 & 0.841 & 2.274 & 1.447 & 7.237 & 4.461 & 4.180 & 2.980 \\
\hline 17.11.2011 & 0.222 & 0.246 & 0.428 & 0.188 & 2.699 & 2.204 & 4.444 & 4.237 & 1.850 & 2.380 \\
\hline 30.11 .2011 & 0.185 & 0.228 & 0.158 & 0.098 & 1.387 & 1.882 & 3.720 & 3.617 & 0.680 & 0.080 \\
\hline 30.12 .2011 & 0.223 & 0.210 & 0.248 & 0.293 & 0.322 & 0.520 & 0.991 & 1.114 & 1.250 & 0.780 \\
\hline 15.02 .2012 & 0.026 & 0.011 & 1.683 & 1.629 & 0.413 & 0.103 & 1.685 & 3.569 & 1.060 & 1.240 \\
\hline 23.03 .2012 & 0.142 & 0.110 & 1.768 & 1.538 & 2.790 & 1.033 & 41.837 & 4.065 & 0.090 & 0.480 \\
\hline 09.05.2012 & 0.007 & 0.010 & 1.731 & 1.752 & 0.827 & 1.240 & 7.931 & 8.129 & 0.980 & 0.990 \\
\hline 01.06 .2012 & 0.017 & 0.099 & 0.756 & 0.981 & 0.103 & 3.617 & 18.242 & 16.457 & 0.430 & 0.180 \\
\hline 09.07.2012 & 0.417 & 0.471 & 1.634 & 0.884 & 8.474 & 5.477 & 9.914 & N/A & 1.380 & 1.450 \\
\hline
\end{tabular}

(N/A: not available)

Table 2. Temporal variations and vertical distribution of $\mathrm{NO}_{2}^{-}+\mathrm{NO}_{3}^{-}, \mathrm{NH}_{4}, \mathrm{PO}_{4}, \mathrm{TP}$ and $\mathrm{SiO}_{2}$ in Station 2 between 30 September 2011 and $09 \mathrm{July}_{2012}$

\begin{tabular}{|c|c|c|c|c|c|c|c|c|c|c|c|c|c|c|c|}
\hline \multirow{2}{*}{ Date } & \multicolumn{3}{|c|}{$\mathrm{NO}_{2}^{-}+\mathrm{NO}_{3}^{-}\left(\mu \mathrm{mol} \mathrm{L}{ }^{-1}\right)$} & \multicolumn{3}{|c|}{$\mathrm{NH}_{4}\left(\mu \mathrm{mol} \mathrm{L}{ }^{-1}\right)$} & \multicolumn{3}{|c|}{$\mathrm{PO}_{4}\left(\mu \mathrm{mol} \mathrm{L}{ }^{-1}\right)$} & \multicolumn{2}{|c|}{ TP $\left(\mu \mathrm{mol} \mathrm{L}{ }^{-1}\right)$} & \multicolumn{4}{|c|}{$\mathrm{SiO}_{2}\left(\mu \mathrm{mol} \mathrm{L}{ }^{-1}\right)$} \\
\hline & Surf. & $15 \mathrm{~m}$ & $30 \mathrm{~m}$ & Surf. & $15 \mathrm{~m}$ & $30 \mathrm{~m}$ & Surf. & $15 \mathrm{~m}$ & $30 \mathrm{~m}$ & Surf. & $15 \mathrm{~m}$ & $30 \mathrm{~m}$ & Surf. & $15 \mathrm{~m}$ & $30 \mathrm{~m}$ \\
\hline 30.09 .2011 & 0.128 & 0.021 & 0.010 & 0.021 & 1.533 & 1.254 & 0.103 & 2.067 & 2.584 & 2.677 & 2.578 & 10.013 & 0.550 & 2.330 & 2.070 \\
\hline 13.10 .2011 & 0.039 & 0.034 & 0.031 & 0.209 & 0.670 & 1.024 & 0.413 & 1.240 & 1.033 & 3.172 & 1.388 & 3.470 & 1.230 & 2.110 & 1.760 \\
\hline 21.10 .2011 & 0.019 & 0.012 & 0.169 & 59 & 1.356 & 1.018 & & 1.240 & 2.790 & & 4.065 & & 2.920 & & 3.790 \\
\hline 17.11 .2011 & 0.224 & 0.218 & & & & & & & & & & & & & 2.840 \\
\hline 30.11 .2011 & 0.168 & 0.217 & 0.283 & 0.090 & 0.180 & 35 & 82 & 1.560 & 1.461 & 5.477 & 3.720 & 4.0 & 1.010 & 0.720 & 1.630 \\
\hline 30.12 .2011 & 0.217 & 0.217 & 0.245 & 0.150 & 0.105 & & & 0.619 & 0.718 & 1.164 & 0.941 & 1.040 & 1.440 & 0.630 & 1.130 \\
\hline 23.03 .2012 & 0.018 & 0.016 & 0.005 & 1.506 & 1.774 & 1.972 & 50 & 0.413 & 0.517 & 4.560 & 2.181 & 4.065 & 0.900 & 2.660 & 0.600 \\
\hline 09.05 .2012 & 0.111 & 0.001 & 0.001 & 1.720 & 2.261 & 1.908 & 0.103 & & 5.750 & 11.599 & 2.067 & 7.441 & 0.830 & 2.100 & 0.280 \\
\hline 01.06 .2012 & 0.061 & 0.049 & 0.052 & 1.334 & 0.697 & 1.195 & 1.137 & 0.930 & 4.031 & 9.418 & 19.431 & 28.255 & 1.030 & 0.250 & 0.830 \\
\hline 09.07 .2012 & 0.428 & 0.405 & 0.517 & 1.174 & 1.785 & 2.653 & 2.280 & 2.274 & 0.991 & 7.751 & N/A & 12.918 & 1.270 & 1.490 & 1.990 \\
\hline
\end{tabular}

(N/A: not available)

Table 3. Temporal variations and vertical distribution of $\mathrm{NO}_{2}^{-}+\mathrm{NO}_{3}^{-} . \mathrm{NH}_{4}, \mathrm{PO}_{4}, \mathrm{TP}$ and $\mathrm{SiO}_{2}$ in Station 3 between 30 September and 09 July 2012.

\begin{tabular}{|c|c|c|c|c|c|c|c|c|c|c|c|c|c|c|c|}
\hline \multirow[t]{2}{*}{ Date } & \multicolumn{3}{|c|}{$\begin{array}{l}\mathrm{NO}_{2}^{-}+\mathrm{NO}_{3}^{-} \\
\left(\mu \mathrm{mol} \mathrm{L}{ }^{-1}\right)\end{array}$} & \multicolumn{3}{|c|}{$\mathrm{NH}_{4}\left(\mu \mathrm{mol} \mathrm{L} \mathbf{L}^{-1}\right)$} & \multicolumn{3}{|c|}{$\left.\mathrm{PO}_{4}(\mu \mathrm{mol} \mathrm{L})^{-1}\right)$} & \multicolumn{3}{|c|}{ TP $\left(\mu \mathrm{mol} \mathrm{L} \mathrm{L}^{-1}\right)$} & \multicolumn{3}{|c|}{$\mathrm{SiO}_{2}\left(\mu \mathrm{mol} \mathrm{L}^{-1}\right)$} \\
\hline & Surf. & $15 \mathrm{~m}$ & 30m & Surf. & $15 \mathrm{~m}$ & 30m & Surf. & $15 \mathrm{~m}$ & $30 \mathrm{~m}$ & Surf. & $15 \mathrm{~m}$ & $30 \mathrm{~m}$ & Surf. & $15 \mathrm{~m}$ & $30 \mathrm{~m}$ \\
\hline 30.09 .2011 & 0.012 & 0.025 & 0.083 & 1.200 & 0.016 & 1.136 & 0.413 & 0.620 & 0.827 & 1.289 & 0.892 & 1.884 & 2.110 & 1.590 & 1.700 \\
\hline 13.10 .2011 & 0.354 & 0.047 & 0.007 & 0.654 & 1.367 & 1.581 & 0.930 & 1.137 & 1.344 & 3.073 & 2.974 & 2.578 & 1.190 & 1.980 & 2.270 \\
\hline 21.10 .2011 & 0.093 & 0.011 & 0.055 & 1.163 & 0.713 & 1.286 & 3.100 & 4.547 & 5.994 & 8.724 & 7.634 & 8.526 & 3.90 & 4.250 & 4.570 \\
\hline 17.11.2011 & 0.248 & 0.237 & 0.229 & 0.120 & 0.315 & 0.450 & 1.860 & 1.436 & 2.130 & 2.625 & 2.480 & 4.444 & 2.200 & 1.610 & 0.680 \\
\hline 30.11 .2011 & 0.339 & 0.279 & 0.315 & 0.180 & 0.600 & 0.105 & 3.393 & 0.619 & 0.892 & 4.134 & 4.547 & 6.718 & 2.450 & 0.940 & 0.820 \\
\hline 30.12 .2011 & 0.306 & 0.265 & 0.259 & 0.188 & 0.300 & 0.083 & 0.644 & 0.892 & 0.520 & 2.130 & 1.313 & 2.799 & 1.440 & 0.630 & 1.130 \\
\hline 15.02 .2012 & 0.058 & 0.014 & 0.010 & 1.586 & 1.881 & 1.736 & 0.517 & 0.310 & 0.723 & 5.056 & 0.991 & 5.906 & 0.740 & 1.690 & 0.817 \\
\hline 23.03 .2012 & 0.032 & 0.020 & 0.022 & 1.238 & 1.892 & 1.929 & 0.207 & 0.207 & 5.994 & 11.302 & 18.142 & 12.293 & 1.410 & 1.215 & 1.250 \\
\hline 09.05 .2012 & 0.021 & 0.011 & 0.044 & 1.827 & 1.179 & 0.965 & 7.441 & 1.785 & 0.103 & 9.517 & 2.274 & 0.991 & 0.280 & 1.180 & 1.160 \\
\hline 01.06 .2012 & 0.033 & N/A & 0.072 & 1.152 & $\mathrm{~N} / \mathrm{A}$ & 1.233 & 0.413 & N/A & 2.997 & 29.940 & N/A & 25.181 & 2.510 & N/A & 0.100 \\
\hline 09.07 .2012 & 0.560 & 0.445 & 0.419 & 1.425 & 2.090 & 1.174 & 0.198 & 5.581 & 0.496 & 2.894 & 11.599 & 6.511 & 0.980 & 1.770 & 1.280 \\
\hline
\end{tabular}

(N/A: not available)

Table 4. Acceptable limits for various physico-chemical water quality parameters for aquaculture and data recorded in the present study.

\begin{tabular}{llll}
\hline Parameter & Acceptable Condition & Present Study & \\
\hline & (Bregnballe, 2015) & S1 & S2 \\
\hline $\mathrm{pH}$ & $6.5-7.5$ & $8.25-8.50$ & $8.25-8.50$ \\
Temperature $\left({ }^{\circ} \mathrm{C}\right)$ & species specific & $11-23$ & $8.00-8.50$ \\
$\mathrm{O}_{2}\left(\% / \mathrm{mg} \mathrm{L}^{-1}\right)$ & $70-100$ & $70-100 / 7-11$ & $11-23$ \\
\hline
\end{tabular}




\begin{tabular}{lllll}
\hline Parameter & Acceptable Condition & Present Study & & \\
\hline & (Bregnballe, 2015) & S1 & S2 & S3 \\
\hline $\mathrm{NH}_{4}\left(\mathrm{mg} \mathrm{L}^{-1}\right)$ & $0-2.5$ (pH influenced) & $0.0005-0.04$ & $0.0004-0.05$ & $0.0003-0.04$ \\
$\mathrm{NO}_{2}\left(\mathrm{mg} \mathrm{L}^{-1}\right)$ & $0-0.5$ & & & \\
$\mathrm{NO}_{3}\left(\mathrm{mg} \mathrm{L}^{-1}\right)$ & $100-200$ & $0.0003-0.02^{*}$ & $0.0001-0.03^{*}$ & $0.0004-0.04^{*}$ \\
$\mathrm{PO}_{4}\left(\mathrm{mg} \mathrm{L}^{-1}\right)$ & $1-20$ & $0.0095-0.804$ & $0.0389-0.216$ & $0.0098-0.707$ \\
\hline
\end{tabular}

S1: station 1; S2: station 2; S3: station 3

${ }^{*}\left[\mathrm{NO}_{2}+\mathrm{NO}_{3}\left(\mathrm{mg} \mathrm{L}^{-1}\right)\right]$

Unit conversions for ammonium nitrogen $\left(\mathrm{NH}_{4}-\mathrm{N}\right): 1 \mu \mathrm{g} \mathrm{NH} \mathrm{N}_{4}^{-1}=0.055437 \mu \mathrm{mol} \mathrm{NH}_{4} \mathrm{~L}^{-1}$;

for nitrite nitrogen $\left(\mathrm{NO}_{2}-\mathrm{N}\right)+$ nitrate nitrogen $\left(\mathrm{NO}_{3}-\mathrm{N}\right): 1 \mu \mathrm{g} \mathrm{NO} \mathrm{N}_{3} \mathrm{~L}^{-1}=0.016128 \mu \mathrm{mol} \mathrm{NO} \mathrm{N}_{3} \mathrm{~L}^{-1}$;

for phosphate phosphorus $\left(\mathrm{PO}_{4}-\mathrm{P}\right): 1 \mu \mathrm{g} \mathrm{PO}_{4} \mathrm{~L}^{-1}=0.010529 \mu \mathrm{mol} \mathrm{PO}_{4} \mathrm{~L}^{-1}$

The results of TSS and chl- $a$ concentration can be seen in Figure 9, 10 and 11 for Stations 1, 2 and 3, respectively. TSS varied among sampling depths between 0.06 and $10.40 \mathrm{mg} \mathrm{L}^{-}$ 1 . Chl- $a$ concentration, which can be considered as a primary production indicator, ranged between $0.143-2.633 \mu \mathrm{g} \mathrm{L}^{-1}$, $0.055-1.519 \mu \mathrm{g} \mathrm{L}^{-1}$, and $0.110-2.288 \mu \mathrm{g} \mathrm{L}{ }^{-1}$, at the surface, at $15 \mathrm{~m}$, and at $30 \mathrm{~m}$ depth, respectively.
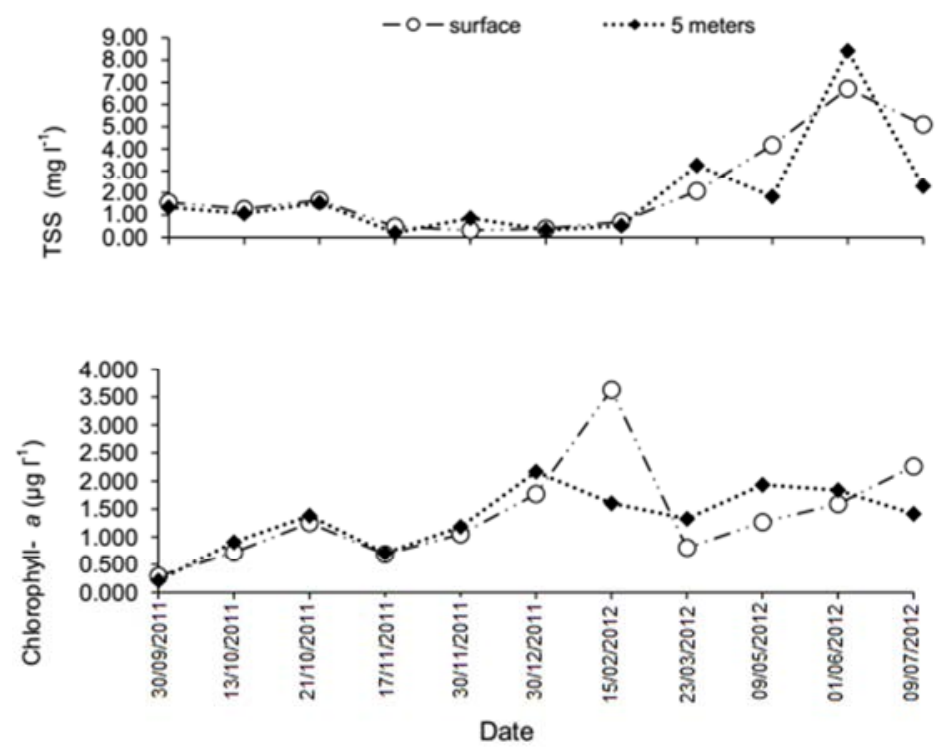

Figure 9. Temporal variations and vertical distribution of TSS and chlorophyll-a at Station 1 between 30 September 2011 and 09 July 2012.
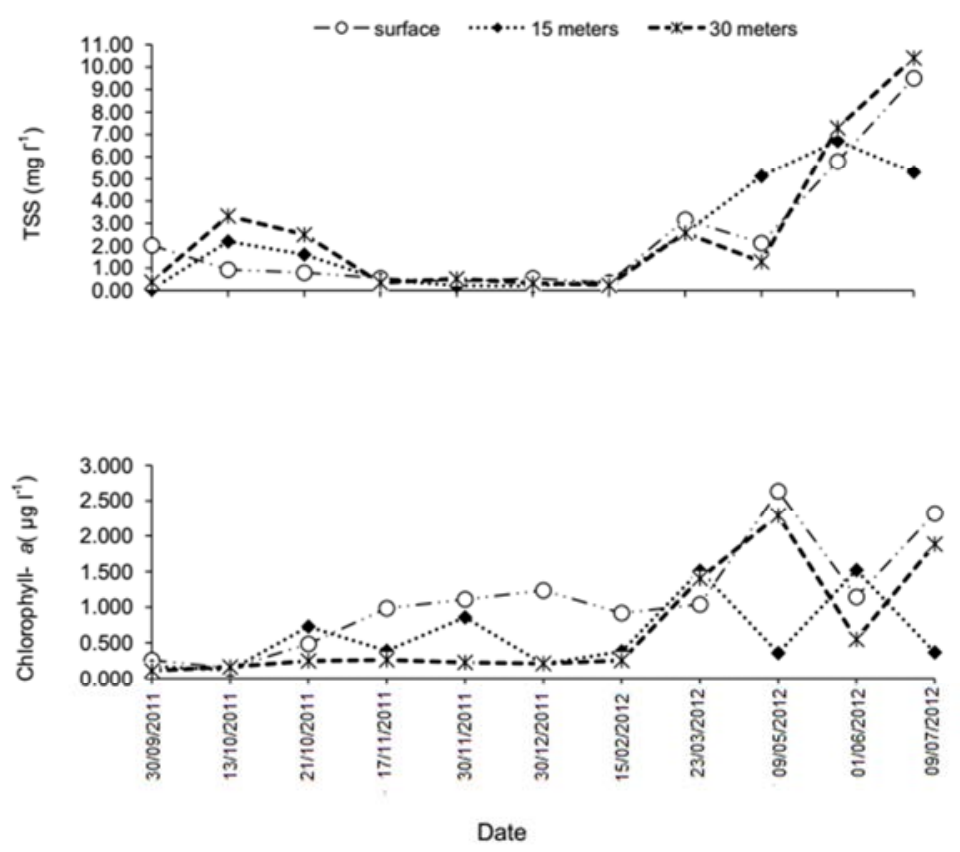

Figure 10. Temporal variations and vertical distribution of TSS and chlorophyll-a at Station 2 between 30 September 2011 and 09 July 2012. 

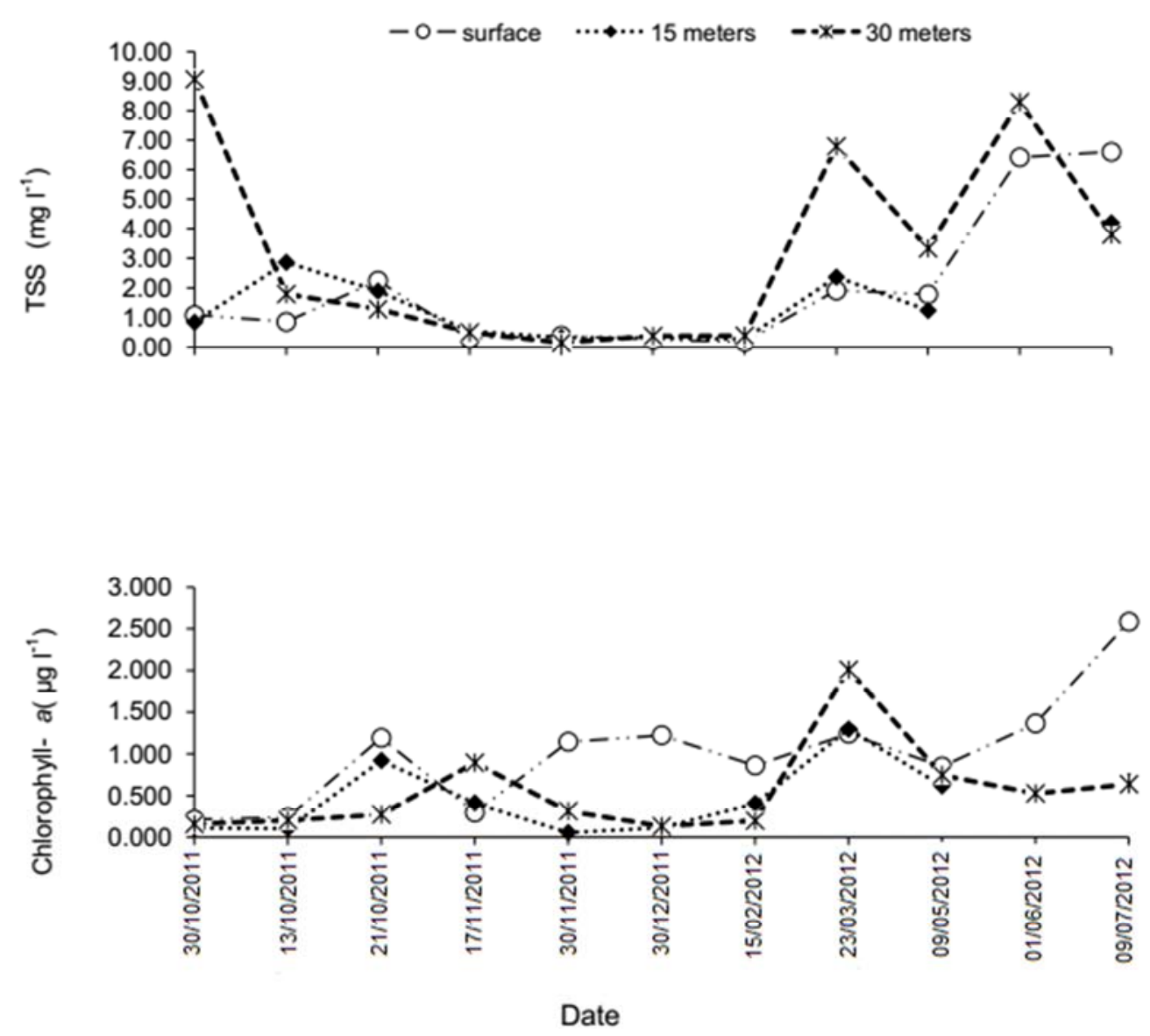

Figure 11. Temporal variations and vertical distribution of TSS and chlorophyll-a at Station 3 between 30 September 2011 and 09 July 2012.

According to the Spearman's rank correlation TSS was positively correlated with $\mathrm{NH}_{4}$ in all stations (Table 5). TSS had a strong positive correlation with TP in the net pens
(Station 1) (Table 4). Chl- $a$ was negatively correlated with $\mathrm{SiO}_{2}$ in Station 2 (Table 5) while there was a positive correlation between Chl- $a$ and TP in Station 3 (Table 5).

Table 5. Spearman's rank correlation between Chl-a, TSS, TP, $\mathrm{PO}_{4}, \mathrm{SiO}_{2}, \mathrm{NH}_{4}, \mathrm{NO}_{2}+\mathrm{NO}_{3}$. Data were depth integrated through the water column.

\begin{tabular}{|c|c|c|c|c|c|c|c|c|}
\hline & & $\begin{array}{l}\text { TP } \\
\left(\mu \mathrm{mol} \mathrm{L} \mathrm{L}^{-1}\right)\end{array}$ & 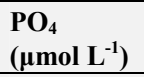 & $\begin{array}{l}\mathrm{SiO}_{2} \\
\left(\mu \mathrm{mol} \mathrm{L}^{-1}\right)\end{array}$ & $\begin{array}{l}\mathrm{NH}_{4} \\
\left(\mu \mathrm{mol} \mathrm{L}^{-1}\right)\end{array}$ & $\begin{array}{l}\mathrm{NO}_{2}+\mathrm{NO}_{3} \\
\left.(\mu \mathrm{mol} \mathrm{L})^{-1}\right) \\
\end{array}$ & $\begin{array}{l}\text { TSS } \\
\left(\mathrm{mg} \mathrm{L}^{-1}\right)\end{array}$ & $\begin{array}{l}\text { chl- } a \\
\left(\mu g \mathrm{~L}^{-1}\right)\end{array}$ \\
\hline \multicolumn{9}{|c|}{ Station 1 - net cage } \\
\hline \multirow{3}{*}{ TSS $\left(\mathrm{mg} \mathrm{L}^{-1}\right)$} & Correlation Coefficient & $.845^{* *}$ & .053 & -.270 & $.444^{*}$ & -.212 & 1.000 & .222 \\
\hline & Sig. (2-tailed) & .000 & .816 & .223 & .038 & .344 & & .321 \\
\hline & $\mathrm{N}$ & 21 & 22 & 22 & 22 & 22 & 22 & 22 \\
\hline \multirow{3}{*}{$\begin{array}{l}\text { Chl- } a \\
\left(\mu \mathrm{g} \mathrm{L}^{-1}\right)\end{array}$} & Correlation Coefficient & .040 & -.039 & -.233 & .383 & -.175 & .222 & 1.000 \\
\hline & Sig. (2-tailed) & .862 & .865 & .296 & .078 & .437 & .321 & \\
\hline & $\mathrm{N}$ & 21 & 22 & 22 & 22 & 22 & 22 & 22 \\
\hline \multicolumn{9}{|c|}{ Station 2 - downstream area } \\
\hline \multirow{3}{*}{$\mathrm{TSS}\left(\mathrm{mg} \mathrm{L}^{-1}\right)$} & Correlation Coefficient & .320 & .169 & -.037 & $.375^{*}$ & -.002 & 1.000 & $.430^{*}$ \\
\hline & Sig. (2-tailed) & .074 & .346 & .838 & .031 & .993 & & .013 \\
\hline & $\mathrm{N}$ & 32 & 33 & 33 & 33 & 33 & 33 & 33 \\
\hline \multirow{3}{*}{$\begin{array}{l}\text { Chl- } a \\
\left(\mu \mathrm{g} \mathrm{L}^{-1}\right)\end{array}$} & Correlation Coefficient & .184 & .131 & $-.346^{*}$ & .313 & .054 & $.430^{*}$ & 1.000 \\
\hline & Sig. (2-tailed) & .314 & .467 & .049 & .076 & .767 & .013 & \\
\hline & $\mathrm{N}$ & 32 & 33 & 33 & 33 & 33 & 33 & 33 \\
\hline \multicolumn{9}{|c|}{ Station 3 - upstream area } \\
\hline \multirow{3}{*}{$\mathrm{TSS}\left(\mathrm{mg} \mathrm{L}^{-1}\right)$} & Correlation Coefficient & .349 & .099 & .194 & $.453^{* *}$ & -.110 & 1.000 & .321 \\
\hline & Sig. (2-tailed) & .051 & .590 & .288 & .009 & .548 & & .073 \\
\hline & $\mathrm{N}$ & 32 & 32 & 32 & 32 & 32 & 32 & 32 \\
\hline \multirow{3}{*}{$\begin{array}{l}\text { Chl- } a \\
\left(\mu \mathrm{g} \mathrm{L}^{-1}\right)\end{array}$} & Correlation Coefficient & $.406^{*}$ & .053 & .066 & .289 & .030 & .321 & 1.000 \\
\hline & Sig. (2-tailed) & .021 & .775 & .718 & .108 & .873 & .073 & \\
\hline & $\mathrm{N}$ & 32 & 32 & 32 & 32 & 32 & 32 & 32 \\
\hline
\end{tabular}

*. Correlation is significant at 0.05 level (2-tailed)

**. Correlation is significant at 0.01 level (2-tailed). 
The results indicate that the horizontal water velocity reached $36 \mathrm{cms}^{-1}$ at select times (Figure3). The average water velocity was found to be $22 \mathrm{cms}^{-1}$. A significant drop in water velocity was observed below the 7 meter mark (5 meters below the instrument). Horizontal water velocities approached $0 \mathrm{cms}^{-1}$ between 10 and $15 \mathrm{~m}$ below the surface ( 8 and $13 \mathrm{~m}$ below the instrument). The dominant current direction, shown in Figure 4, was found to be $180^{\circ}$ (South) in the first $13 \mathrm{~m}$ of the water column. Although not plotted, the water velocities had similar water velocities below the pycnocline heading in the opposite direction $\left(045^{\circ}-000^{\circ}\right)$, as expected.

The vertical velocity structure (Figure 5) points out distinctive down-welling at the farm site. The surface water tends to sink towards the bottom with a velocity of $10 \mathrm{cms}^{-1}$ at 2 meters below the surface, and slows as it reaches the pycnocline.

The water quality parameters were found to vary among sampling depths throughout the sampling period. The highest salinity value was measured at $30 \mathrm{~m}$ at Station 3 on 30 November 2011. This was expected as the more dense water from the Aegean Sea moves northward below the less dense surface water directed downward from the Black Sea. Water temperature followed the progression of the seasons. The characteristics of two different water masses could be clearly indicated depending on the temperature and salinity values in the study area of Canakkale Strait. The values recorded for $\mathrm{pH}$ were in the range of standard seawater $\mathrm{pH}$ values [12]. Additionally, salinity, temperature and $\mathrm{pH}$ values were similar to previously obtained environmental parameters, measured in separate studies close to the farm site [13 - 17].

Inorganic nutrient concentrations changed from low to moderate through most of the sampling period. Silicate values were lower than $3 \mathrm{mg} \mathrm{L}-1$ which was well below the optimum value for diatom growth in marine systems. Other than sporadic nutrient input through rain and wind drawn mixing events, analysis of nutrients among sampling depths over time showed that dissolved inorganic nitrogen to phosphorus ratios (DIN/PO4) did not follow the common Redfield Ratio and were lower, suggesting a nitrogen limitation of primary production. Except high TP values on March, May and June samplings, nutrient values were in the range when compared to previously conducted studies in the surrounding area $[16,17]$.

The values recorded for TSS were below the typical threshold for seawater quality measurement of $30 \mathrm{mg} \mathrm{L}-1$, pronounced by [18]. Additionally, these values were lower compared to those reported in previous studies conducted in the vicinity while chlorophyll-a concentrations were similar [14 - 17].

Influence of phytoplankton abundance on TSS in open oceans is considered to be more than in coastal systems where terrestrial sources have the main role on TSS [19]. In this study, it has been observed that starting from March 2012, especially in Stations 2 and 3, phytoplankton abundance controlled the TSS, rather than terrestrial sources (Figs. 10, 11; Table 5).
Strong positive correlation between TSS and TP as well as NH4 may be an indication of the accumulation of unused fish feed or fecal material of fish in the net cage system (Table 5). Negative correlation of chl-a and $\mathrm{SiO}_{2}$ suggested that primary production may become limited by silica.

The study results showed that surface water velocities headed south at a speed of $22 \mathrm{cms}^{-1}$. Down-welling was seen at a speed of $10 \mathrm{cms}^{-1}$. All the water quality parameters, total suspended solids and nutrients were found to be similar between measurement stations, showing no influence of the copper alloy mesh cage and farmed fish on the surrounding water environment.

\section{Conclusion}

Copper alloy meshes become a promising material with improved economic benefits, cleaner cage environment, biofouling resistance as well as improved strength under high-energy offshore environments. The weakest area that has not been studied thoroughly was the environmental monitoring around copper alloy cages in offshore conditions with respect to bio-chemical water parameters. Results in the present study showed that all measurements were consistent with other water quality studies conducted in the area in recent years, showing steady water characteristics of the fish farming site. This study can be considered as an exemplary for other areas having similar exposed environmental conditions with the study site.

\section{Acknowledgements}

This study was funded by the International Copper Association, New York-USA with the project code of "ICA Project No. TEK-1049". Canakkale Onsekiz Mart University, Scientific Research Projects Commission (COMU-BAP) is acknowledged for the support of laboratory equipments used in this study with the Project ID: 256 and Project Code: FAY2014-256.

\section{References}

[1] Yigit, M., Celikkol, B., Gace, L., DeCew, J., Hisar, O., Bulut, M., Yildiz, H., Ozalp, B., Kaya, H., Yilmaz, S., \& Irkin, L. C. (2013). Present state and future expectations of Mediterranean aquaculture: environmental concern and benefits of copper alloy nettings for a sustainable high value aquaculture industry. WAS-Asian pacific, High value aquaculture finfish symposium. Kagoshima University-Japan, 15-18 October 2013.

[2] Grass, G., Rensing, C., \& Solioz, M. (2011). Metallic copper as an antimicrobial surface. Applied Environmental Microbiology, 77(5), 1541-1547.

[3] Gonzalez, E. P., Hurtado, C. F., Gace, L., \& Augsburger, A. (2013). Economic impacts of adopting copper alloy mesh in trout aquaculture: Chilean example. Aquacic Economic Management, 17(1), 71-86. 
[4] Tsukrov, I., Drach, A., DeCew, J., Swift, M. R., \& Celikkol, B. (2011). Characterization of geometry and normal drag coefficients of copper nets. Ocean Engineering, 38, 19791988.

[5] Yigit, M., Celikkol, B., DeCew, J., Bulut, M., Kesbic, O. S., Karga, M., Özalp, B., \& Büyükates, Y. (2012). Copper-alloy mesh in offshore aquaculture systems - A new net material for cage farming in the Southern European seas. In: Abstracts of the 4th Offshore Mariculture Conference, Izmir, 17-19 October 2012.

[6] Bulut, M., Yigit, M., Ergun, S., Kesbic, O. S., Acar, U., Gultepe, N., Karga, M., Yılmaz, S., \& Guroy, D. (2014). Evaluation of dietary protein and lipid requirements of twobanded seabream (Diplodus vulgaris) cultured in a recirculating aquaculture system. Aquaculture International, $22,965-973$.

[7] Ayer, N., Martin, S., Dwyer, R. L., Gace, L., \& Laurin, L. (2016). Environmental performance of copper-alloy net-pens: Life cycle assessment of Atlantic salmon grow-out in copperalloy and nylon net-pens. Aquaculture, 453(1), 93-103.

[8] Parsons, T. R., Maita, Y. \& Lalli, C. M., (1984). A manual of chemical and biological methods for seawater analysis. Pergamon, Oxford.

[9] Clesceri, L. S., Greenberg, A. E., \& Eaton, A. E. (1998). Standard Methods for the Examination of Water and Wastewater. Method 2540 B. (Total Solids). Method 2540 C. (Total Dissolved Solids) and Method 2540 D. (Total Suspended Solids). 20th Edition.

[10] Strickland, J. D. H., \& Parsons, T. R. (1972). A practical handbook of seawater analysis. 2nd ed. Fisheries Research Board of Canada.

[11] Bregnballe J. (2015). A guide to recirculation aquaculture, an introduction to the new environmentally friendly and highly productive closed fish farming systems. Publication of Food and Agriculture Organization of the United Nations (FAO) and EUROFISH International Organisation.

[12] Kocataş, A. (1993). Oseanoloji. Ege Üniveristesi Kitaplar Serisi. Bornova. İzmir. (In Turkish).

[13] Koçum, E. (2005). Çanakkaleboğazında klorofila ve çözünmüş mineral besin elementi miktarlarının analizi. Ekoloji, 14, 1-6. (In Turkish).

[14] Buyukates, Y., \& İnanmaz, Ö. E. (2007). Temporal variations in vertical distribution and occurrence of marine cladocerans in an urbanized harbour. Dardanelles. Turkey. Crustaceana, 80, 1293-1302.

[15] Buyukates, Y., \& İnanmaz, Ö. E. (2009). Cladocerans of an urbanized harbour: effects of environmental parameters on vertical distribution, occurrence, abundance, and seasonal variation. Crustaceana, 82, 543-554.

[16] Buyukates, Y., \& İnanmaz, Ö. E. (2010). The Annual Mesozooplankton Dynamics and influence of environmental parameters in an urbanized harbor (Kepez Harbor-Dardanelles Strait, Turkey). Ekoloji, 19, 60-68.

[17] Odabaşı, S., \& Büyükates, Y. (2009). Daily Variation of chlorophyll-a, environmental parameters and nutrients: Sarıçay creek as an exemplary (Çanakkale, Turkey). Ekoloji, 19, 76-85.

[18] WPCR (Water Pollution Control Regulations). (2012). Official Gazette (ResmiGazete): 31 December 2012, Friday, Issue: 25687 (In Turkish).

[19] Besiktepe, S., Sur, H. I., Ozsoy, E., Latif, M. A., Oguz, T., \& Unluata, U. (1994). The circulation and hydrography of the Marmara Sea. Progress in Oceanography, 34, 285-334. 